\title{
Control of Decoherence and Relaxation by Frequency Modulation of Heat Bath
}

\author{
G.S. Agarwal * \\ Physical Research Laboratory, Navrangpura, Ahmedabad-380 009, India.
}

(May 24, 2022)

\begin{abstract}
We demonstrate in a very general fashion, considerable slowing down of decoherence and relaxation by fast frequency modulation of the system heat bath coupling. The slowing occurs as the decoherence rates are now determined by the spectral components of bath correlations which are shifted due to fast modulation. We present several examples including the slowing down of the heating of a trapped ion, where the system - bath interaction is not necessarily Markovian.
\end{abstract}

PACS No: 03.65 Bz, 03.67a, 5.30-d, 42.50 Md

In recent times the decoherence of a coherent superposition state has acquired a new dimension [1 5 ] because of the requirement of the stability of such a superposition. The stability has been investigated for certain systems. The decoherence rates have been calculated and even measured [6] in the context of Cat like states [7] for the radiation field in a cavity. The decoherence issues are also very significant in the context of quantum computation [8 [10]. Clearly the stability of coherent superpositions requires methods for slowing down the decoherence. Several proposals exist in the literature [11 13]. These involve for example use of a sequence of pulses [12] or engineering of the density of states associated with the reservoir or even changing the reservoir interaction from a single photon to multiphoton (or more generally multiboson) interaction [11,14]. Other proposals involve feedback methods [13]. It may be added that spontaneous emission in many systems is also a cause of decoherence. We now understand reasonably well, how to inhibit spontaneous emission either by manipulating the density of states [15] or by using external fields [16, 17]. The methods based on external fields could be especially useful for slowing down decoherence.

In this letter, we discuss a method based on the frequency modulation [18 20] of the system-heat bath (environment) coupling. We specifically assume large frequency modulation and take the modulation index $m$ to have a value given by $J_{0}(m)=0$. Under these conditions, we demonstrate considerable slowing down of the decay and decoherence rates. We present a physical basis for this slowing down. We present several examples including the heating of a trapped ion. Our method is useful only if the correlation time $\kappa^{-1}$ of the heat bath is larger than the rate of frequency modulation i.e. $\nu^{-1}$. It must be noted that a recent experimental proposal to control decoherence [6(b)] also depends on a coherent coupling with a bath (second single mode cavity) and works under similar conditions [21].

In order to appreciate the basic idea of using frequency modulation we consider a two state system, where the states $|a\rangle$ and $|b\rangle$ are coupled by some field. We also assume that the state $|b\rangle$ decays at the rate $2 \kappa$. This simple model [Fig.1] can describe many physical situations. For example, it can represent an excited atom in a cavity [22] in which the photon leaks out at the rate $2 \kappa$. In this case the states $|a\rangle$ and $|b\rangle$ will correspond to $|e, o\rangle,|g, 1\rangle$, where $|o\rangle$ and $|1\rangle$ represent vacuum and one photon state respectively and where $|e\rangle$ and $|g\rangle$ represent the excited and ground states of the atom. It can also describe a situation where the state $|b\rangle$ could be an excited state coupled to ionization continuum. The probability amplitudes $C_{a}$ and $C_{b}$ for the states $|a\rangle$ and $|b\rangle$ obey the equations

$$
\begin{aligned}
& \dot{C}_{a}=-i g C_{b}, \\
& \dot{C}_{b}=-\kappa C_{b}-i g^{*} C_{a} .
\end{aligned}
$$

We have removed any fast time dependence by working in an appropriate frame. If $\kappa$ is large, then as is well known

$$
\begin{aligned}
\left|C_{a}\right|^{2} & \cong \exp \{-2 \Gamma t\}, \\
\Gamma & =|g|^{2} / \kappa ; \kappa \gg g .
\end{aligned}
$$

The decay of the state $|a\rangle$ arises from the decay of the state $|b\rangle$. In the opposite limit $(\kappa \rightarrow 0)$ one gets oscillatory behavior, which in the cavity context is known as the vacuum field Rabi oscillation. We now consider the effect of a phase modulation $m \sin \nu t$ on the decay of the state $|a\rangle$ : we assume a modulation of the coupling constant

$$
g \rightarrow g \exp \{-i m \sin \nu t\} .
$$

*also at Jawaharlal Nehru Centre for Advanced Scientific Research, Bangalore, India. 
Here $m$ and $\nu$ give respectively, the amplitude and the frequency of the modulation. Equation (1) is no longer amenable to analytical solutions. In Fig. 2, we display the excited state population for different values of $\nu$ and $m$ chosen to be a zero of the Bessel function of order zero

$$
J_{o}(m)=0 .
$$

This choice of $m$ will become clear in the analysis to follow. In Fig. 2 we also show the behavior in the absence of modulation. We observe that under the condition(4) the decay of the excited state population is considerably slowed as the modulation frequency increases. This clearly demonstrates how a frequency modulation can slow down the effects of decay. We thus have a method of controlling relaxation / decay by frequency modulation. We now explain the observed numerical behavior for large $\nu$. Using (1), we can easily derive the following integro-differential equation for the amplitude of the excited state

$$
\dot{C}_{a} \equiv-|g|^{2} e^{-i \Phi(t)} \int_{0}^{t} e^{-\kappa(t-\tau)+i \Phi(\tau)} C_{a}(\tau) d \tau
$$

We use

$$
e^{-i \Phi(t)}=\sum_{l=-\infty}^{+\infty} J_{l}(m) e^{-i l \nu t},
$$

and we assume that (i) $\nu$ is large (ii) $C_{a}(\tau)$ varies slowly with $\tau$ and carry out a long time average denoted by over bar to get

$$
\begin{array}{r}
\frac{\partial}{\partial t}\left(\operatorname{lnC}_{\mathrm{a}}\right) \equiv-|g|^{2} \int_{0}^{t} e^{-\kappa \tau} \overline{e^{-i \Phi(t)} e^{i \Phi(t-\tau)}} d \tau \\
\equiv-|g|^{2} \sum_{p} J_{p}^{2}(m)(\kappa+i p \nu)^{-1}
\end{array}
$$

In order to slow down the decoherence, we need to remove the $\nu=0$ term in (7). This can be achieved by imposing the condition (4) whence (7) reduces to

$$
\frac{\partial}{\partial t}\left(\ln C_{\mathrm{a}}\right) \approx-2|g|^{2} J_{1}^{2}(m)\left(\kappa^{2}+\nu^{2}\right)^{-1} \kappa .
$$

Therefore the decay of the excited state occurs at a modified rate $2 \tilde{\Gamma}$ with

$$
\frac{\tilde{\Gamma}}{\Gamma} \cong J_{1}^{2}(m)\left(\frac{2 \kappa^{2}}{\kappa^{2}+\nu^{2}}\right)
$$

The decay factor (9) agrees very well with the behavior shown in the Fig. 2 for $20 \pi$ as then $\nu \gg \kappa$. The very fast oscillations do not show up on the scale of the Fig. 2. The result (9) can be understood by noting that - (i) the factor in the parenthesis in (9) is just the factor that one would have obtained with a detuned interaction between the states $|a\rangle$ and $|b\rangle$; (ii) the Bessel function represents the strength of the first side band.

We next demonstrate that the above idea applies rather generally. We consider the usual microscopic treatment of the heat bath [23] with the modulation of the system heat bath coupling. For the purpose of illustration, we consider a spin system [raising and lowering operators $S^{+}$and $S^{-}$] interacting say with a dc and ac magnetic field in $\mathrm{Z}$ direction so that the unperturbed Hamiltonian is $\left(\omega_{0}-m \nu \cos \nu t\right) S^{z}$. The energy separation gets modulated - such modulations are routinely used (see e.g. Noel et al [18]). In the interaction picture the interaction with the heat bath can be written as

$$
H_{I}(t)=\left(S^{+} e^{+i \omega_{0} t-i \Phi(t)} R^{-}(t)+\text { H.c. }\right),
$$

where $R^{-}(t)$ is the appropriate operator for the heat bath. As usual 23] we will assume that the coupling of the bath to the system is weak. The heat bath is characterized in terms of the correlation functions:

$$
\begin{aligned}
\left\langle R^{-}(t)\right\rangle & =0, \\
\left\langle R^{+}(t+\tau) R^{-}(t)\right\rangle & =C^{+-}(\tau), \\
\left\langle R^{-}(t+\tau) R^{+}(t)\right\rangle & =C^{-+}(\tau), \\
\left\langle R^{-}(t+\tau) R^{-}(t)\right\rangle & =0 .
\end{aligned}
$$


The Fourier transforms of $C^{+-}$and $C^{-+}$are related via the fluctuation dissipation theorem. We can now do the standard calculation [23] to derive a master equation for the reduced density matrix $\rho$ of the system alone. We quote the result of this calculation.

$$
\begin{aligned}
\frac{\partial \rho}{\partial t}= & -\left(S^{+} S^{-} \rho-S^{-} \rho S^{+}\right) \int_{0}^{t} d \tau C^{-+}(\tau) e^{+i \omega_{0} \tau} e^{-i \Phi(t)} e^{i \Phi(t-\tau)}, \\
& -\left(\rho S^{-} S^{+}-S^{+} \rho S^{-}\right) \int_{0}^{t} d \tau C^{+-}(-\tau) e^{+i \omega_{0} \tau} e^{i \Phi(t-\tau)-i \Phi(t)}, \\
& + \text { terms with subscripts } \pm \rightarrow \mp, \omega_{0} \rightarrow-\omega_{0}, \Phi \rightarrow-\Phi .
\end{aligned}
$$

First of all we note, that if the bath correlations were like delta correlations $C^{-+}(\tau)=2 \delta(\tau) C^{-+}$, then the master equation (12) does not depend on the modulation $\Phi$. Clearly, the bath correlation time $\tau_{c}$ has to be at least of the order of the time associated with the modulation. Under the fundamental condition (4), the time average in (12) can be approximated by

$$
\overline{e^{-i \Phi(t)+i \Phi(t-\tau)}} \cong 2 J_{1}^{2}(m) \cos \nu \tau
$$

and then (12) reduces to

$$
\begin{array}{r}
\frac{\partial \rho}{\partial t} \equiv-2\left(S^{+} S^{-} \rho-S^{-} \rho S^{+}\right) \int_{0}^{\infty} d \tau C^{-+}(\tau) e^{+i \omega_{0} \tau} \cos \nu \tau J_{1}^{2}(m), \\
-2\left(\rho S^{-} S^{+}-S^{+} \rho S^{-}\right) \int_{0}^{\infty} d \tau C^{+-}(-\tau) e^{+i \omega_{0} \tau} \cos \nu \tau J_{1}^{2}(m), \\
+ \text { terms with } \pm \rightarrow \mp, \omega_{0} \rightarrow-\omega_{0} .
\end{array}
$$

The standard master equation corresponds to the limits $\nu \rightarrow 0, J_{1}^{2} \rightarrow 1$. It is clear that if $\nu$ is large enough compared to frequency scale of $C^{-+}(\tau) e^{i \omega_{0} \tau}$ then the real part of the integral in (14) will be approximately zero and decoherence effectively does not exist. In particular, if $C^{-+}(\tau)=C_{0}^{-+} e^{-\kappa \tau-i \omega \tau}, C^{+-}(-\tau)=C_{0}^{-+} e^{-\kappa \tau-i \omega \tau}$, then

$$
\begin{aligned}
\frac{\partial \rho}{\partial t} & =-\frac{2(\kappa-i \Delta) J_{1}^{2}(m)}{(\kappa-i \Delta)^{2}+\nu^{2}}\left\{C_{0}^{-+}\left(S^{+} S^{-} \rho-S^{-} \rho S^{+}\right)\right. \\
& \left.+C_{0}^{+-}\left(\rho S^{-} S^{+}-S^{+} \rho S^{-}\right)\right\}+ \text {c.c.. }, \Delta=\left(\omega_{0}-\omega\right) .
\end{aligned}
$$

Clearly, the relaxation coefficients in the master equation are modified by factors like (9). Hence the relaxation is much slower. In particular, the relaxation of the coherence $\left\langle S^{ \pm}\right\rangle$will be on a much longer time scale. For large $\nu$ compared to $\kappa$ and $\Delta$, the relaxation time is very large.

We next consider the application of the above ideas to the decoherence of an ion in a trap. This is important in many applications of ion traps such as in connection with the production of Cat states and in quantum computation. In particular, we consider the possibility of reducing the heating of the ground state of trapped ions. In a recent letter, James [10] has considered a model for heating produced by a stochastic field $\mathrm{E}(\mathrm{t})$. In terms of the annihilation and creation operators $a, a^{\dagger}$ associated with the ionic motion the heating is described by the Hamiltonian

$$
H_{1}=i \hbar\left[u(t) a^{\dagger}-u^{*}(t) a\right]
$$

where $u(t)=i Z E(t) e^{i \omega_{0} t} / \sqrt{2 M \hbar \omega_{0}}$. The field $E(t)$ is a Gaussian stochastic process. The time scale of the stochastic field is taken to be comparable to the time scale of the ionic motion. Hence this model is outside the usual markovian limit. We now consider the effect of an external modulation so that effectively, $u(t) \rightarrow u(t) e^{-i \Phi(t)}$. Following James' work the fidelity $\mathrm{F}(\mathrm{t})$ of the ground state is given by

$$
\begin{gathered}
F(t)=\left[1+2\left\langle|v(t)|^{2}\right\rangle+\left\langle|v(t)|^{2}\right\rangle^{2}-\left|\left\langle v^{2}(t)\right\rangle\right|^{2}\right]^{-1 / 2}, \\
v(t)=\frac{i Z}{\sqrt{2 M \hbar \omega_{0}}} \int_{0}^{t} E\left(t^{\prime}\right) e^{-i \Phi\left(t^{\prime}\right)+i \omega_{0} t^{\prime}} d t^{\prime} .
\end{gathered}
$$

The mean values in (17) can be obtained from (18) by assuming exponential correlation for $E(t)$ : 


$$
\begin{aligned}
\left\langle u(t) u^{*}\left(t^{\prime}\right)\right\rangle & =\frac{\Omega^{2}}{2} e^{-\kappa\left|t-t^{\prime}\right|} \\
\left\langle|v|^{2}\right\rangle & =\frac{\Omega^{2}}{2} \sum_{-\infty}^{+\infty} \sum J_{n}(m) J_{p}(m) I\left(\omega_{0}-n \nu ;-\omega_{0}+p \nu\right) \\
\left\langle v^{2}\right\rangle & =-\frac{\Omega^{2}}{2} \sum_{-\infty}^{+\infty} \sum J_{n}(m) J_{p}(m) I\left(\omega_{0}-n \nu ; \omega_{0}-p \nu\right)
\end{aligned}
$$

where the integral $I\left(\omega_{\alpha}, \omega_{\beta}\right)$ is found to be

$$
\begin{aligned}
I\left(\omega_{\alpha}, \omega_{\beta}\right) & =\left(i \omega_{\alpha}+i \omega_{\beta}\right)^{-1}\left[\left(\kappa+i \omega_{\beta}\right)^{-1} e^{i\left(\omega_{\alpha}+\omega_{\beta}\right) t}-\left(\kappa-i \omega_{\alpha}\right)^{-1}\right] \\
& +\left(i \omega_{\alpha}-\kappa\right)^{-1}\left(-i \omega_{\beta}-\kappa\right)^{-1} e^{i \omega_{\alpha} t-\kappa t} \\
& + \text { terms with } \alpha \Leftrightarrow \beta .
\end{aligned}
$$

Note that $\omega_{\alpha}+\omega_{\beta}$ can vanish in which case, a limiting procedure leads to

$$
I\left(\omega_{\alpha},-\omega_{\alpha}\right)=\left(\kappa-i \omega_{\alpha}\right)^{-1} t+\left(i \omega_{\alpha}-\kappa\right)^{-2}\left(e^{i \omega_{\alpha} t-\kappa t}-1\right)+\text { c.c. }
$$

We show the fidelity factor $F$ in Fig. 3, both in the absence and presence of the modulation. We choose a parameter domain in which fidelity was being degraded rather fast. Clearly, if we assume large frequency modulation and condition (4), then as the figure shows, there is considerable improvement in the fidelity under frequency modulation of the stochastic field $\mathrm{E}(\mathrm{t})$ responsible for heating the trapped ion.

In conclusion, we have shown how the appropriate modulation of the system-heat bath interaction can slow down the decay as well as the decoherence to a very large extent. This happens as generally, the decoherence is determined by the spectral components of the bath correlation functions. If system-bath interaction is modulated, then the decoherence is determined by the spectral components, which are shifted by the multiples of the modulation frequency. If the modulation frequency is large compared to the width of the bath correlations, then we would get much smaller decoherence rate. Finally, note that we have a method to control the effects of decoherence since the modulation depth and frequency can be varied.

The author thanks Sunish Menon for the plots.

[1] See e.g. recent books and reviews [2]

[2] M. Namiki, S. Pascazio and H. Nakazato, "Decoherence and Quantum Measurements", (Word Scientific, Singapore, 1997).

[3] "Quantum Coherence and Decoherence" eds. K. Fujikawa and Y.A. Ono, (North Holland, Amsterdam, 1996).

[4] E. Joos in D. Giulini et al., "Decoherence and the Appearance of a Classical World in Quantum Theory" (Springer, Berlin, 1996) p.35; W.H. Zurek, Prog. Theoret. Phys. 89, 281 (1993); Physics Today 44, 36 (1991); a recent work [G.S.Agarwal, Phys. Rev.A in press] discusses diagonalization in coherent state basis.

[5] V. Buzek and P.L. Knight in "Progress in Optics", ed. E. Wolf (North Holland, Amsterdam, 1995) Vol.34, p.l; M.S. Kim and V. Buzek, J. Mod. Opt. 39, 1609 (1992).

[6] (a) M. Brune, E. Hagley, J. Dreyer, X. Maitre, A. Maali, C. Wunderlich, J.M. Raimond and S. Haroche, Phys. Rev. Lett. 77, 4887 (1996); (b) J.M. Raimond, M. Brune and S. Haroche Phys. Rev. Lett. 79, 1964 (1997).

[7] J.J. Slosser and G.J. Milburn, Phys. Rev. Lett. 75, 418 (1995); C.C. Gerry, Opt. Commun. 91, 247 (1992); C. Monroe, D.M. Meekhof, B.E. Knig and D.J. Wineland, Science 272, 1131 (1996); D. Leibfried, D.M. Meekhof, B.E. Knig, C. Monroe, W.M. Itano and D.J. Wineland, Phys. Rev. Lett. 77, 4281 (1996); R.L. deMatos Filho and W. Vogel, Phys. Rev. Lett. 76, 608 (1996).

[8] M.B. Plenio and P.L. Knight, Proc. Roy. Soc. London A453, 2017 (1997); G.M. Palma, K.A. Suominen and A.K. Ekert, Proc. Roy.Soc. London Ser. A452, 567 (1996); D.P. Divincenzo, Science 270, 255 (1995).

[9] M. Murao and P.L. Knight, Phys. Rev. A58, 663 (1998); S. Schneider and G.J. Milburn, Phys. Rev. A57, 3748 (1998).

[10] D.F.V. James, Phys. Rev. Lett. 81, 317 (1998).

[11] J.F. Poyatos, J.I. Cirac and P. Zoller, Phys. Rev. Lett. 77, 4738 (1996).

[12] Use of a sequence of pulses can slow down decoherence : L. Viola and S. Lloyd, Phys. Rev. A58, 2733 (1998); L. Viola, E. Knill and S. Lloyd, to be published. 
[13] D. Vitali, P.Tombesi and G.J. Milburn, Phys. Rev. Lett. 79, 2442 (1997); J. Mod. Optics, 44, 2033 (1997); D.B. Horoshko and S. Ya.Kilin, Phys. Rev. Lett. 78, 840 (1997); J. Mod. Optics 44, 2043, (1997).

[14] G.S. Agarwal, Phys. Rev. Lett. 57, 827 (1986); G.C. Gerry and E.E. Hach III, Phys. Lett. A174, 185 (1993).

[15] E. Yablonovitch, Phys. Rev. Lett. 59, 2059 (1987); S. John and T. Quang, Phys. Rev. A50, 1764 (1994).

[16] G.S. Agarwal, W. Lange and H. Walther, Phys. Rev. A48, 4555 (1993); T.W. Mossberg and M. Lewenstein, in "Cavity Quantum Electrodynamics" ed. P.R. Berman (Academic, New York, 1994)p.171.

[17] External fields have generally been shown to lead to quenching of noise effects - G. Vemuri, K.V. Vasavada and G.S. Agarwal, Phys. Rev. A50, 2599 (1994).

[18] Earlier works have shown how the fast frequency modulation in coherently driven systems can lead to the inhibition of the transition: G.S. Agarwal and W. Harshawardhan, Phys. Rev. A50, R4465 (1994); W. Harshawardhan and G.S. Agarwal, Phys. Rev. A55, 2165 (1997). For experimental verification of some of these developments see M.W. Noel, W.M. Griffith and T.F. Gallagher, Phys. Rev. A58, 2265 (1998). For related theoretical developments see references [20].

[19] S. Raghavan, V.M. Kenkre, D.H. Dunlap, A.R. Bishop and M.I. Salkola, Phys. Rev. A54, R1781 (1996); P.K. Lam and C.M. Savage, Phys. Rev. A50, 3500 (1994); P.M. Radmore, S.Tarzi and K.L. Tong, J. Mod. Opt. 42, 2213 (1995).

[20] V.N. Ostrovsky and H. Nakamura, J. Phys. A30, 6939 (1997); F. Grossmann, T. Dittrich, P. Jung and P. Hanggi, Phys. Rev. Lett. 67, 516 (1991); M. Wagner, Phys. Rev. A51, 798 (1995).

[21] For the experimental proposal of ref. [6b]; $\tau \ll \kappa^{-1}$ ( $\kappa$ related to the cavity leakage time) and thus $\frac{\Omega_{c} \tau}{2 \pi} \ll \frac{\Omega_{c}}{2 \pi \kappa}$ which further requires that $\Omega_{c} / \kappa$ be large [cf. Fig. 2 of ref. [6(b)]].

[22] P. Meystre in "Progress in Optics", ed. E. Wolf, (North Holland, Amsterdam, (1992)), vol.30, p263.

[23] F. Bloch, Phys. Rev. 102, 104 (1956); ibid 105, 1206 (1957).

FIG. 1. Schematic representation of a generic two level system with lower level decay into a bath. A strong modulation of the coupling slows the decay of the upper level.

FIG. 2. The Population in the excited state $\left|C_{a}(t)\right|^{2}$ (Eqs.(1),(3)) as a function of $t$ for different values of the modulation frequency and for $\kappa=10 \mathrm{~g}$. The modulation index is chosen to be the fifth zero of (4). The curves from top to bottom are for $\frac{\nu}{\pi g}=20,5,0.5$ and 0.05 . The curve for $m=0$ is hardly distinguishable from the curve for $\frac{\nu}{\pi g}=0.05$.

FIG. 3. Fidelity (17) or the heating of an ion in the trap for two different values of the modulation frequency $\nu=5,3$. The dashed curve gives the result in the absence of the modulation. Parameters are chosen as $\omega_{0} / \kappa=1 ; \Omega[$ defined by Eq.(19) $]=\sqrt{2} \omega_{0}$. The wiggles arise from the periodic modulation. 

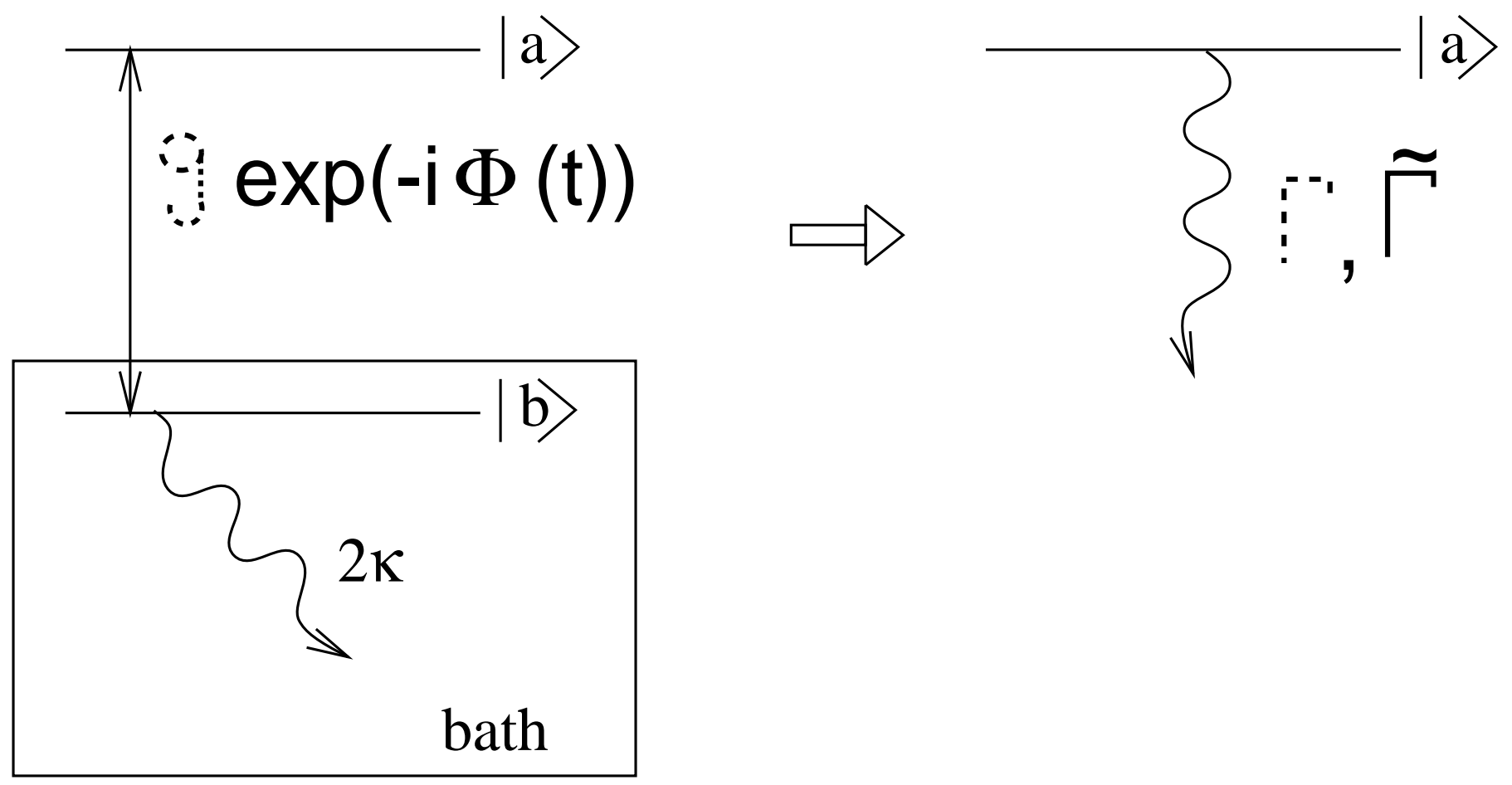


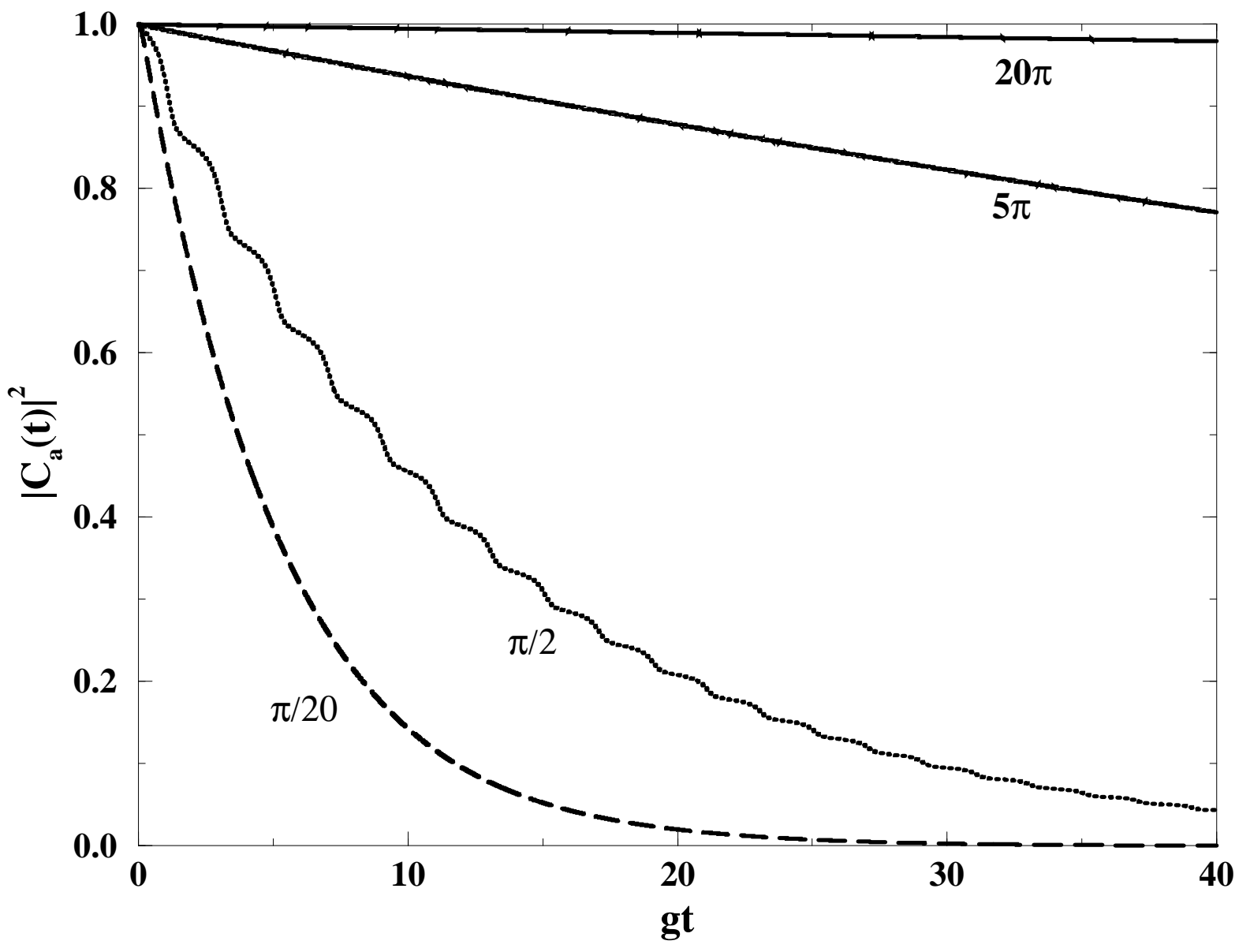




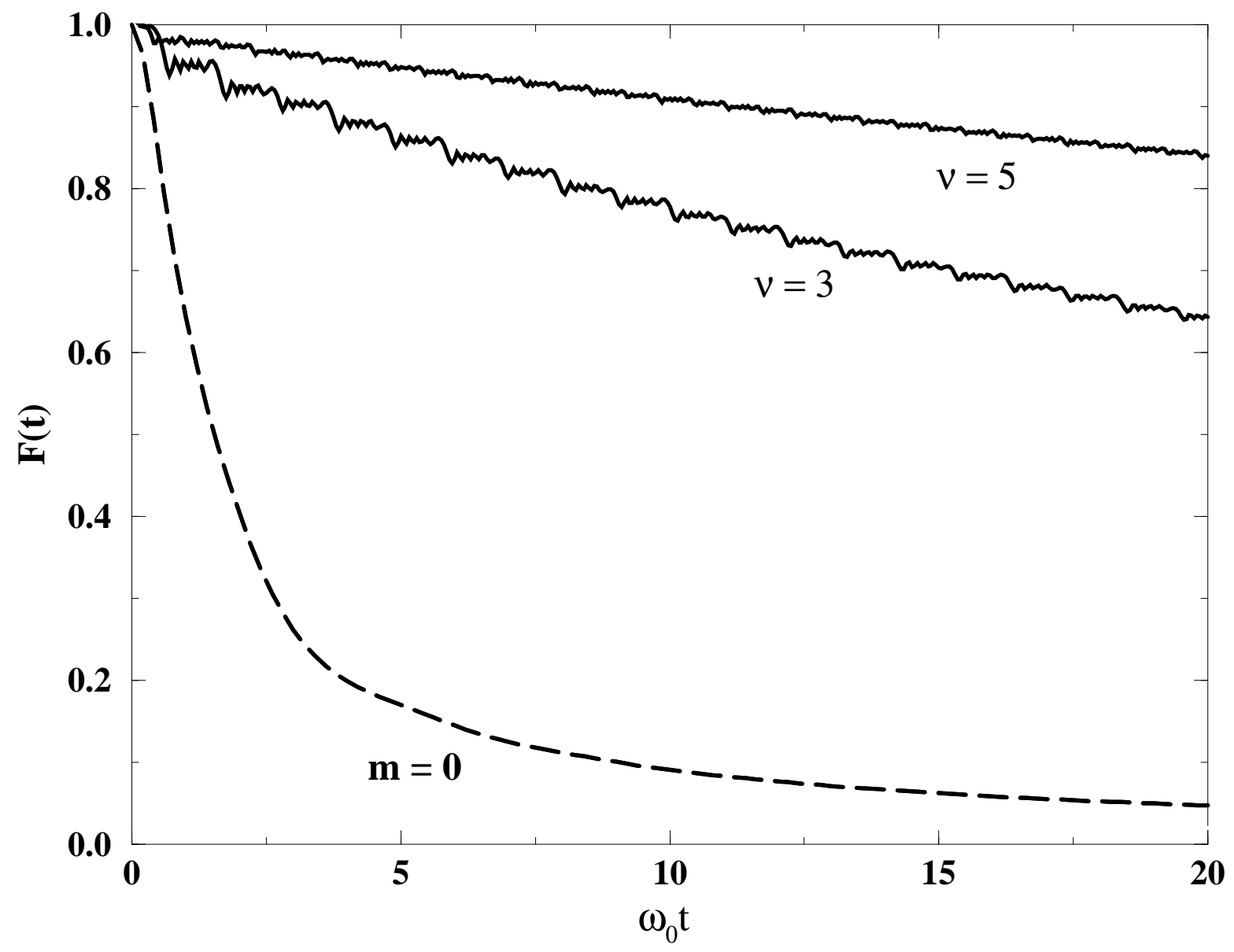

